\title{
CsPrx25, a class III peroxidase in Citrus sinensis, confers resistance to citrus bacterial canker through the maintenance of ROS homeostasis and cell wall lignification
}

\author{
Qiang Li ${ }^{1}$, Xiujuan Qin', Jingjing Qi ${ }^{1}$, Wanfu Dou', Christophe Dunand ${ }^{2}$, Shanchun Chen ${ }^{1}$ and Yongrui He $\mathrm{H}^{1,3}$
}

\begin{abstract}
Citrus bacterial canker (CBC) results from Xanthomonas citri subsp. citri (XcC) infection and poses a grave threat to citrus production. Class III peroxidases (CIII Prxs) are key proteins to the environmental adaptation of citrus plants to a range of exogenous pathogens, but the role of CIII Prxs during plant resistance to $C B C$ is poorly defined. Herein, we explored the role of CsPrx25 and its contribution to plant defenses in molecular detail. Based on the expression analysis, CsPrx 25 was identified as an apoplast-localized protein that is differentially regulated by Xcc infection, salicylic acid, and methyl jasmone acid in the CBC-susceptible variety Wanjincheng (C. sinensis) and the CBC-resistant variety Calamondin ( $C$. madurensis). Transgenic Wanjincheng plants overexpressing CsPrx25 were generated, and these transgenic plants exhibited significantly increased CBC resistance compared with the WT plants. In addition, the CsPrx25-overexpressing plants displayed altered reactive oxygen species (ROS) homeostasis accompanied by enhanced $\mathrm{H}_{2} \mathrm{O}_{2}$ levels, which led to stronger hypersensitivity responses during Xcc infection. Moreover, the overexpression of CsPrx25 enhanced lignification as an apoplastic barrier for Xcc infection. Taken together, the results highlight how CsPrx25-mediated ROS homeostasis reconstruction and cell wall lignification can enhance the resistance of sweet orange to CBC.
\end{abstract}

\section{Introduction}

Plants possess an intricate repertoire of cell-based defense systems to maintain their resistance to potentially harmful pathogens ${ }^{1,2}$. As an immediate pathogen recognition response, oxidative bursts produced in apoplasts induce reactive oxygen species (ROS), including superoxide $\left(\mathrm{O}_{2}{ }^{-{ }^{-}}\right)$ and $\mathrm{H}_{2} \mathrm{O}_{2}$, as a first line of defense ${ }^{3}$. The current models of plant responses include ROS and other radicals as catalysts of covalent cell-wall modifications ${ }^{4}$, as signals for cell-death reactions ${ }^{5,6}$ and as regulators of resistance-associated genes $^{7,8}$. In plants, high concentrations of ROS act to

\footnotetext{
Correspondence: Shanchun Chen (chenshanchun@cric.cn) or

Yongrui He (heyongrui@cric.cn)

${ }^{1}$ Citrus Research Institute, Southwest University/Chinese Academy of Agricultural Sciences, Chongqing 400712, China

'Laboratoire de Recherche en Sciences Végétales, Université de Toulouse,

CNRS, UPS, Auzeville-Tolosane 31320, France

Full list of author information is available at the end of the article
}

strengthen the cell wall and inhibit pathogen growth, which results in the enhancement of host resistance to pathogens via hypersensitive responses (HRs) and the modulation of gene expression via signaling molecules ${ }^{9,10}$. However, high accumulation of ROS can be toxic to plant cells by inhibiting plant growth and development ${ }^{2}$. Thus, ROS homeostasis needs to be maintained by antioxidant compounds and enzymes $^{11}$. In plant cells, ROS are produced by NADPH oxidase resident at the cell surface, class III peroxidases (CIII Prxs, or POD) and their associated pathways, including photosynthesis, photorespiration, and respiration ${ }^{12,13}$. In addition, the ROS scavengers superoxide dismutase (SOD), catalase (CAT), and glutathione s-transferase (GST) cooperate with ROS producers to maintain ROS homeostasis ${ }^{14}$. Moreover, antioxidant enzyme activities and ROS homeostasis are regulated by important plant hormones, including jasmonic acid (JA) and salicylic acid (SA) ${ }^{15-17}$. 
CIII Prxs are heme-binding proteins that are ubiquitously expressed in all plants and comprise large multigene families ${ }^{18-21}$. For example, a total of 73 CIII Prxs are present in Arabidopsis thaliana ${ }^{22,23}$, and 138, 374, 93, 94 and 72 have been found in Oryza sativa ${ }^{24}$, Triticum aestivum $^{25}$, Populus trichocarpa ${ }^{26}$, Pyrus bretschneideri ${ }^{27}$ and Citrus sinensis ${ }^{28}$, respectively. CIII Prxs regulate the loosening of cell walls, lignification and suberization ${ }^{29-32}$ and participate in ROS and RNS metabolism during abiotic and biotic stress responses ${ }^{33-35}$. CIII Prxs are key to the innate resistance of many plants to both fungal and bacterial pathogens and mediate both passive and active defense mechanisms ${ }^{6,36,37}$, and the efficiency of this mediation determines their susceptibility to pathogenic infections ${ }^{38}$. Rapid ROS production is one such exemplar defense strategy that leads to $\mathrm{O}_{2}{ }^{--}$generation and $\mathrm{H}_{2} \mathrm{O}_{2}$ production in apoplasts. $\mathrm{H}_{2} \mathrm{O}_{2}$ is tightly regulated by CIII Prxs as both producers and scavengers depending on whether the enzyme participates in peroxidative cycles and hydroxylic cycles, respectively ${ }^{12,13}$. In French bean and tobacco plants, apoplastic CIII Prxs produce ROS and act as catalysts for covalent cell-wall modifications ${ }^{4}$ and cell death regulators ${ }^{6}$. Based on these functions of CIII Prxs, an increasing number of studies have identified links between this enzyme and pathogen attack and have improved host resistance due to CIII Prxs. Radwan and colleagues reported that bean yellow mosaic virus infection leads to increased levels of monodihydroascorbate (MDA) and $\mathrm{H}_{2} \mathrm{O}_{2}$ in Vicia faba leaves ${ }^{39}$. Enhanced CIII Prx and SOD activities have also been observed in leaves infected by yellow mosaic virus, which suggests that enzymatic antioxidants regulate ROS generation in response to pathogen infection ${ }^{39}$. Increasing the expression of a peroxidase in plants can effectively increase the resistance of the plants to disease. For example, the overexpression of $\operatorname{HvPr} \times 40^{40}$ and $\operatorname{TaPr} \times 10^{39,41}$ leads to higher levels of resistance to Blumeria graminis (wheat powdery mildew) in wheat (T. aestivum).

Xanthomonas citri subsp. citri $(X c c)$ pathogen is the causative agent of citrus bacterial canker (CBC), a known cause of citrus yield losses in an array of citrus-producing regions ${ }^{42,43}$. In our previous studies of the citrus transcriptomes induced by Xcc, we found that CIII Prxs were differentially expressed and explored the relationship between $\mathrm{CBC}$ and CIII Prxs, and our results revealed CsPrx25 as a potential gene for improving CBC resistance ${ }^{28}$. Here, we performed both a structural and functional characterization of CsPrx25. We also developed transgenic sweet orange overexpressing CsPrx25 that displayed enhanced tolerance to $\mathrm{CBC}$ due to ROS homeostasis accompanied by high levels of $\mathrm{H}_{2} \mathrm{O}_{2}$ and high lignification of the apoplastic barrier. We herein describe the utility of transgenic plants overexpressing CsPrx25 for enhancing CBC resistance.

\section{Results}

\section{CsPrx25 encodes a CIII Prx in citrus}

We amplified and sequenced the complete transcript of CsPrx 25 using cDNA from Wanjincheng leaves as the PCR template. The primary sequences were searched in PeroxiScan, which is built in RedoxiBase ${ }^{44,45}$. The findings revealed that CsPrx25 belonged to the CIII Prx family (PeroxiScan accession: PS52045), a subgroup of nonanimal peroxidases (PeroxiScan accession: PS50873). The CsPrx 25 sequence was further analysed by the Blast tool built in RedoxiBase and $\mathrm{CAP}^{46}$, and the results revealed that CsPrx 25 was clustered with the CIII Prxs sequence ID 8898 in RedoxiBase and Cs3g21730 in CAP due to 100 and $98 \%$ sequence similarities, respectively. CsPrx 25 is a 344-residue CIII Prx (molecular weight: $38.06 \mathrm{kD}$; isoelectric point: 8.55$)$ present on chromosome 3 of $C$. sinensis (Fig. 1a) that possesses two introns (1515 bp and $659 \mathrm{bp}$, respectively) (Fig. 1b). The $\mathrm{N}$-terminus of CsPrx 25 contains a signal peptide of 27 residues that is required for correct trafficking to the apoplast. Throughout the sequence, eight cysteine residues were detected $(\mathrm{C} 1-\mathrm{C} 8)$ (Fig. 1C), and these form a total of four disulfide bonds (DB) that maintain thermal stability. These 4-DB structures are common to almost all plant CIII Prxs and impart distinction from ascorbate and other plant peroxidases ${ }^{47}$. The three-dimensional (3D) structures also showed that the cysteines that form disulfide bonds are close to each other (Fig. 1d). To study the evolutive scenario of CIII Prxs between organisms, the phylogeny of CIII Prxs orthologs was assessed, and close relationships between CsPrx25 and AtPrx12 were found (Fig. 1e).

\section{CsPrx25 is an apoplast-localized protein that is induced by XcC and phytohormones}

To elucidate the localization of CsPrx25, software predictions and transient expression systems were investigated. CELLO V2.5 displayed extracellular loci values of 2.46, which were larger than other loci (Supplementary Table S1). The signal peptide detected by SignalP V4.0 suggests that CsPrx25, as most of the CIII Prxs, is extracellular. To validate these predictions, the transient expression of CsPrx 25 was assessed with $35 S:: C s \operatorname{Pr} x 25-$ GFP (Fig. 2a). Relative to the controls, both cytoplasmic and nuclear fluorescence were observed before and after plasmolysis (Fig. 2b). In epidermal onion cells, CsPrx25GFP showed robust cell surface expression (Fig. 2c), confirming that CsPrx25 localizes to apoplasts.

Pathogens and phytohormones can mediate gene expression changes that occur in response to plant disease $^{48,49}$. In Calamondin, CsPrx 25 was upregulated, and maximal expression ( 5-fold) was observed at $36 \mathrm{hpi}$. In contrast, Wanjincheng CsPrx25 showed little-to-no expressional changes in response to Xcc infection (Fig. $2 d$ ). To detect the effect of drought during in vitro 


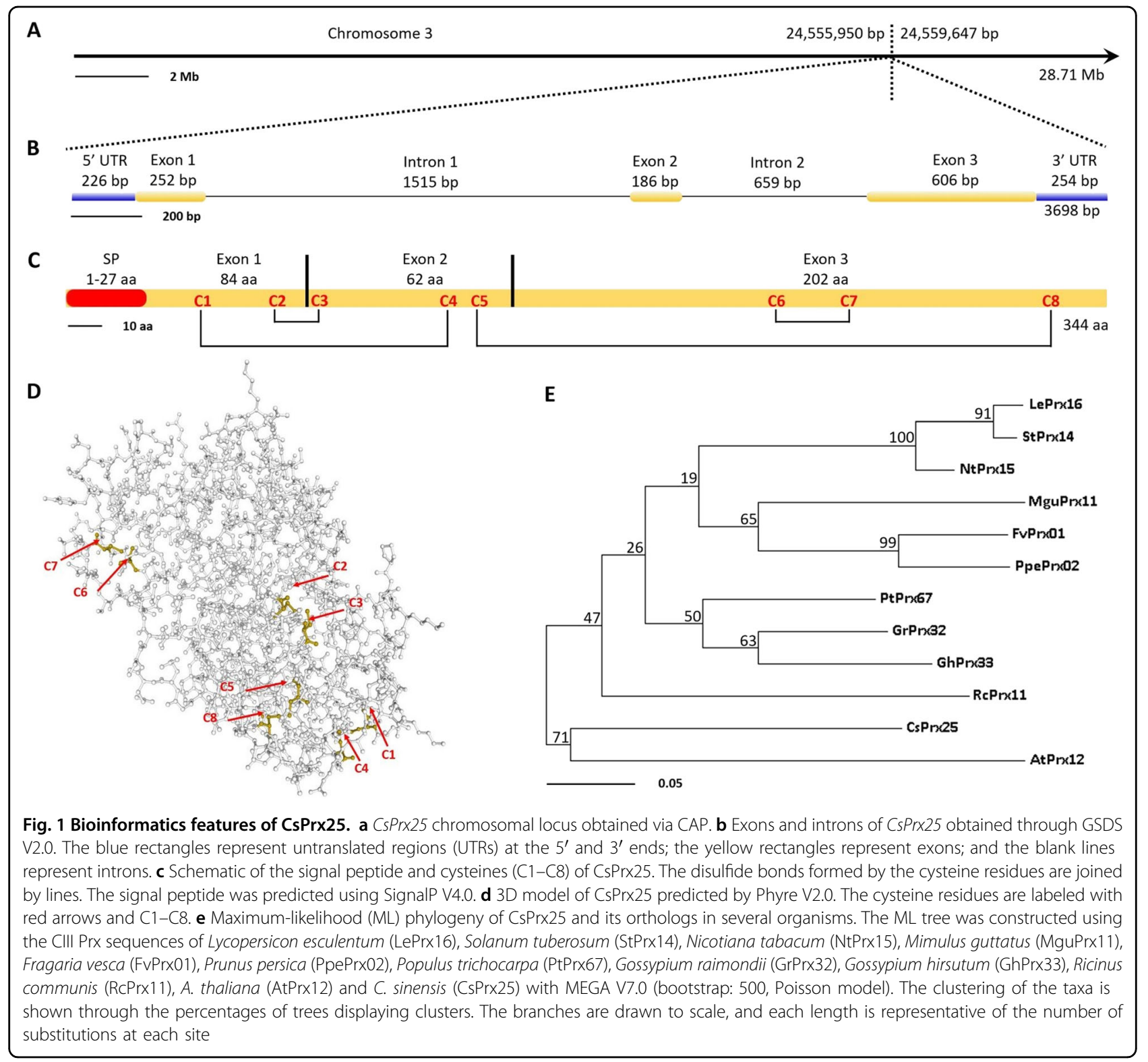

inoculation, we tested the inducibility of CsPrx 25 under drought stress. The results indicated that CsPrx 25 was hardly induced by drought in both varieties, which indicated that it was specifically induced by Xcc (Supplementary Fig. S1). CsPrx 25 is therefore likely to represent an $X c c$ resistance gene. To reveal the molecular mechanisms through which CsPrx25 mediates disease resistance, CsPrx 25 transcripts were assessed in SA- and MeJA-treated leaves. The expression of CsPrx 25 rapidly increased in Calamondin in response to SA. In contrast, CsPrx25 expression was downregulated in Wanjincheng (Fig. 2e). The expression of CsPrx 25 induced by MeJA increased and then decreased over time in both Wanjincheng and Calamondin, and the times to maximal expression in these varieties was different (Wangjincheng:
24 hpt vs Calamondin: 6 hpt) (Fig. 2f). The different expression patterns of CsPrx 25 induced by phytohormones indicate the different roles of $C s P r x 25$ in disease resistance signaling in Calamondin and Wanjincheng.

\section{CsPrx25 overexpression in sweet orange induces resistance to $\mathrm{CBC}$}

Transgenic citrus constructs overexpressing CsPrx 25 were used to fully dissect the role of CsPrx25 during Xcc resistance. CsPrx 25 was overexpressed using exogenous expression plasmids driven by the $35 \mathrm{~S}$ promoter (Fig. 3a). The generation of four CsPrx25-overexpressing plants 1-4 (OE1-OE4) that successfully integrated CsPrx 25 was confirmed by qRT-PCR, GUS assay and Southern blot. Through PCR, we detected an 1874-bp fragment that was 


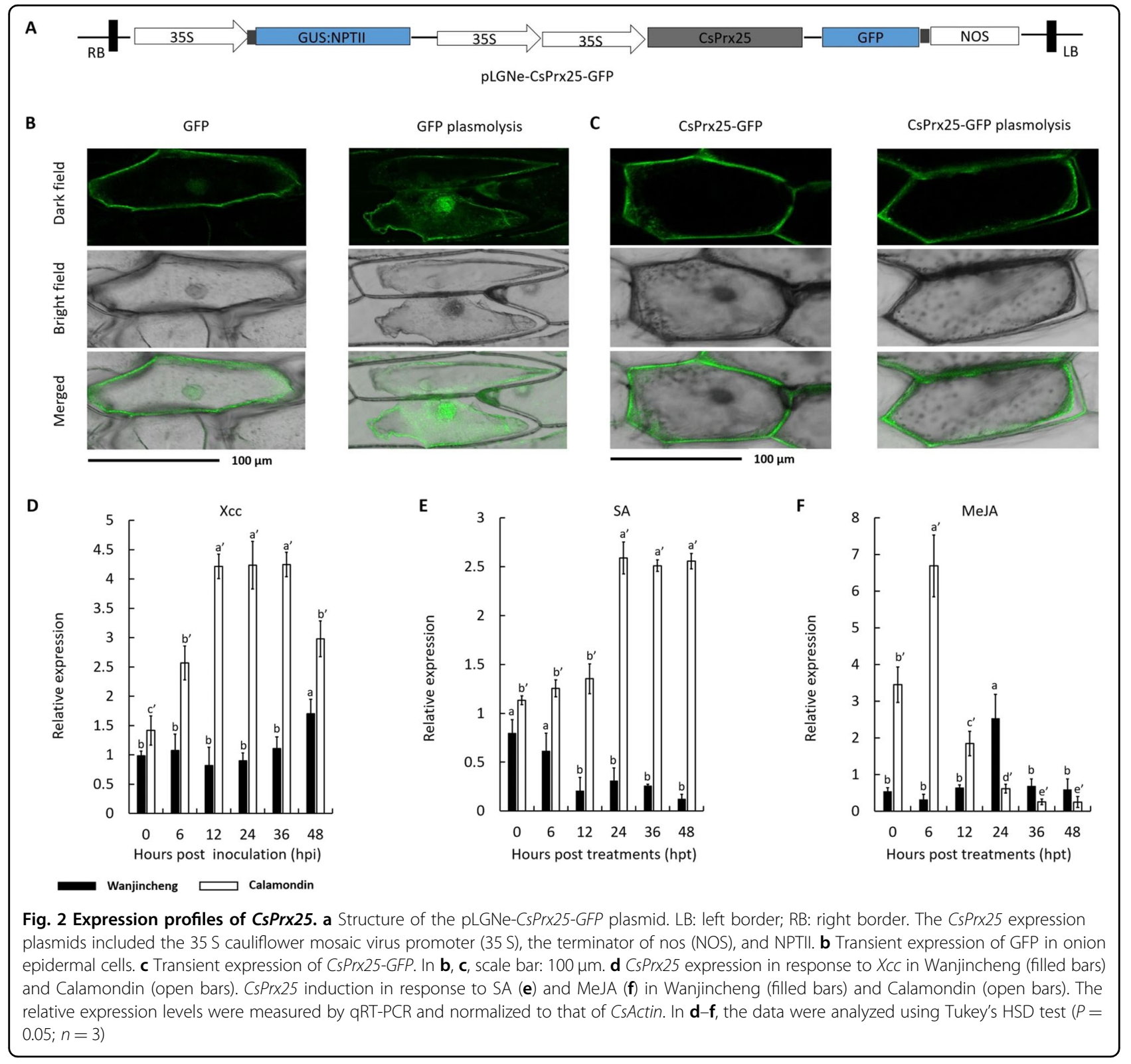

not present in the wild-type (WT) lines (Fig. 3b), and the GUS assay revealed blue color on the periphery of the leaf discs (Fig. 3c). As determined by Southern blot, OE1 and OE2 contain two copies of CsPrx25, and OE3 and OE4 harbor only one copy (Fig. 3d). We confirmed that all lines expressed high levels of CsPrx25 (550-fold, 589-fold, 401-fold and 395-fold of the WT levels, respectively) by qRT-PCR analysis (Fig. 3e). According to the Southern blot assay, a certain positive correlation exists between copy number and expression (Fig. 3d). With respect to phenotypes, the four transgenic lines showed normal growth rates compared with the WT lines (Fig. 3f). Acupuncture is an effective method for quantitatively assessing the resistance to $\mathrm{CBC}$ and can be used to accurately quantify $\mathrm{CBC}$ resistance, which would allow the assessment and comparison of resistance between varieties $^{50,51}$. To assess the $\mathrm{CBC}$ resistance of $C s \operatorname{Pr} x 25-\mathrm{OE}$ plants, in vitro assays were performed with acupuncture inoculation at $10 \mathrm{dpi}$. Smaller lesion sizes, which are indicative of less-severe symptoms, were observed in the OE leaves compared with the WT leaves (Fig. 3g). This finding suggested that $X c c$ pustules are reduced by CsPrx 25 overexpression, and OE2 showed the highest levels of resistance. Compared with the WT plants, OE2 showed smaller lesions (45.8\% of the WT levels), OE1 exhibited comparable lesions $(47.0 \%$ of the WT levels), and OE3 and OE4 displayed larger lesions (65.8\% and $68.8 \%$ of the WT levels) (Fig. 3h). The disease severity 


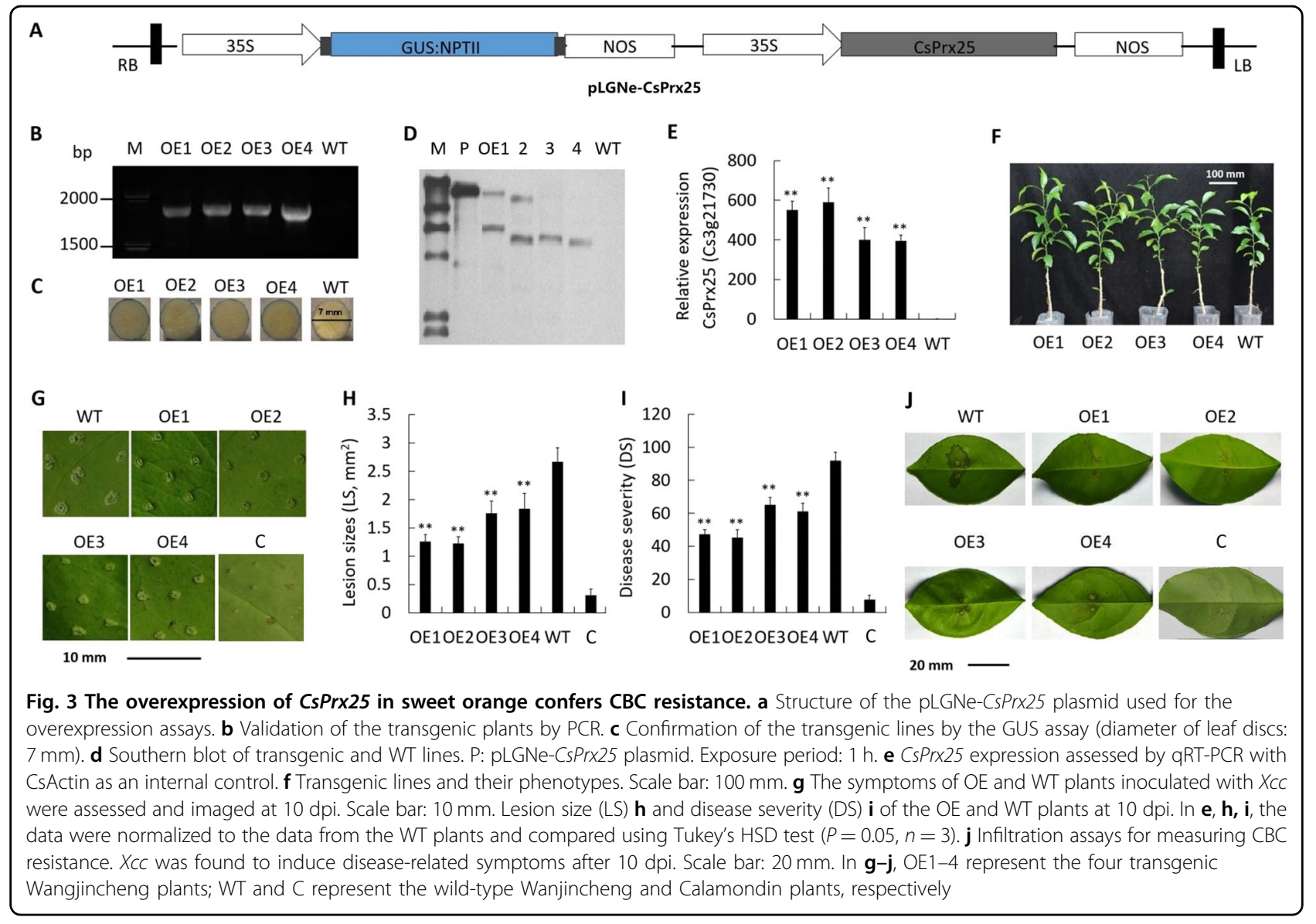

decreased by $29.2 \%$ (OE3) to $50.7 \%$ (OE2) in the $\mathrm{OE}$ plants compared with WT plants (Fig. 3i). Using infiltration assays after $10 \mathrm{dpi}$, symptoms of canker (including pustules) were observed in then WT lines, but these symptoms were markedly reduced in the OE plants (Fig. 3j). We therefore conclude that CsPrx 25 overexpression enhances $X c c$ resistance in the OE transgenic citrus lines.

\section{CsPrx25 overexpression modulates the enzymatic antioxidant system}

Plants possess a well-developed ROS homeostasis enzymatic system that efficiently regulates the ROS levels, and this system includes CIII Prx, SOD, CAT and $\mathrm{GST}^{38,52}$. To assess the changes in the antioxidant system following the induction of CsPrx25-mediated resistance to $X c c$, the antioxidant activity in transgenic lines in these lines was compared with that in the WT plants at $12 \mathrm{hpi}$. OE plants with higher resistance (OE1 and OE2) to CBC were selected for analysis. The activities of both CIII Prx and SOD were upregulated by CsPrx 25 overexpression (Fig. 4a, b). The overexpression of CsPrx25 conferred antioxidant defenses and led to the induction by Xcc infection. In contrast to CIII Prx and SOD, the activities of CAT in OE plants were downregulated, and the Xcc- induced profiles were altered compared with those observed in the WT plants (Fig. 4c). In contrast to SOD, CIII Prx and CAT, the overexpression of CsPrx25 did not affect the activity of GST compared with that found in the WT plants (Fig. 4d).

\section{CsPrx25 overexpression establishes ROS homeostasis to confer a more sensitive HR to Xcc infection}

In response to pathogen infection, ROS production intricately controls many responses, including apoptotic cell death and oxidative damage ${ }^{53,54}$. To confirm the involvement of ROS homeostasis in CsPrx25-mediated $X c c$ resistance, the levels of $\mathrm{H}_{2} \mathrm{O}_{2}$ and $\mathrm{O}_{2}{ }^{-}$in WT vs. CsPrx 25-OE lines were assessed. We observed higher levels of $\mathrm{H}_{2} \mathrm{O}_{2}$ in the OE lines. Of interest, Xcc infection did not significant change the levels of $\mathrm{H}_{2} \mathrm{O}_{2}$ in WT plants but increased these levels in the OE lines (Fig. 5a). This finding suggested that CsPrx 25 overexpression not only increased the levels of $\mathrm{H}_{2} \mathrm{O}_{2}$ but also reversed the inducible patterns of $\mathrm{H}_{2} \mathrm{O}_{2}$ during $X c c$ infection. The levels of $\mathrm{O}_{2}{ }^{-}$also increased in response to CsPrx25 overexpression (Fig. 5b). The cell membrane is first affected by lipid peroxidation, and MDA is the final product ${ }^{55}$. A spectroscopic analysis of the transgenic and WT plants 

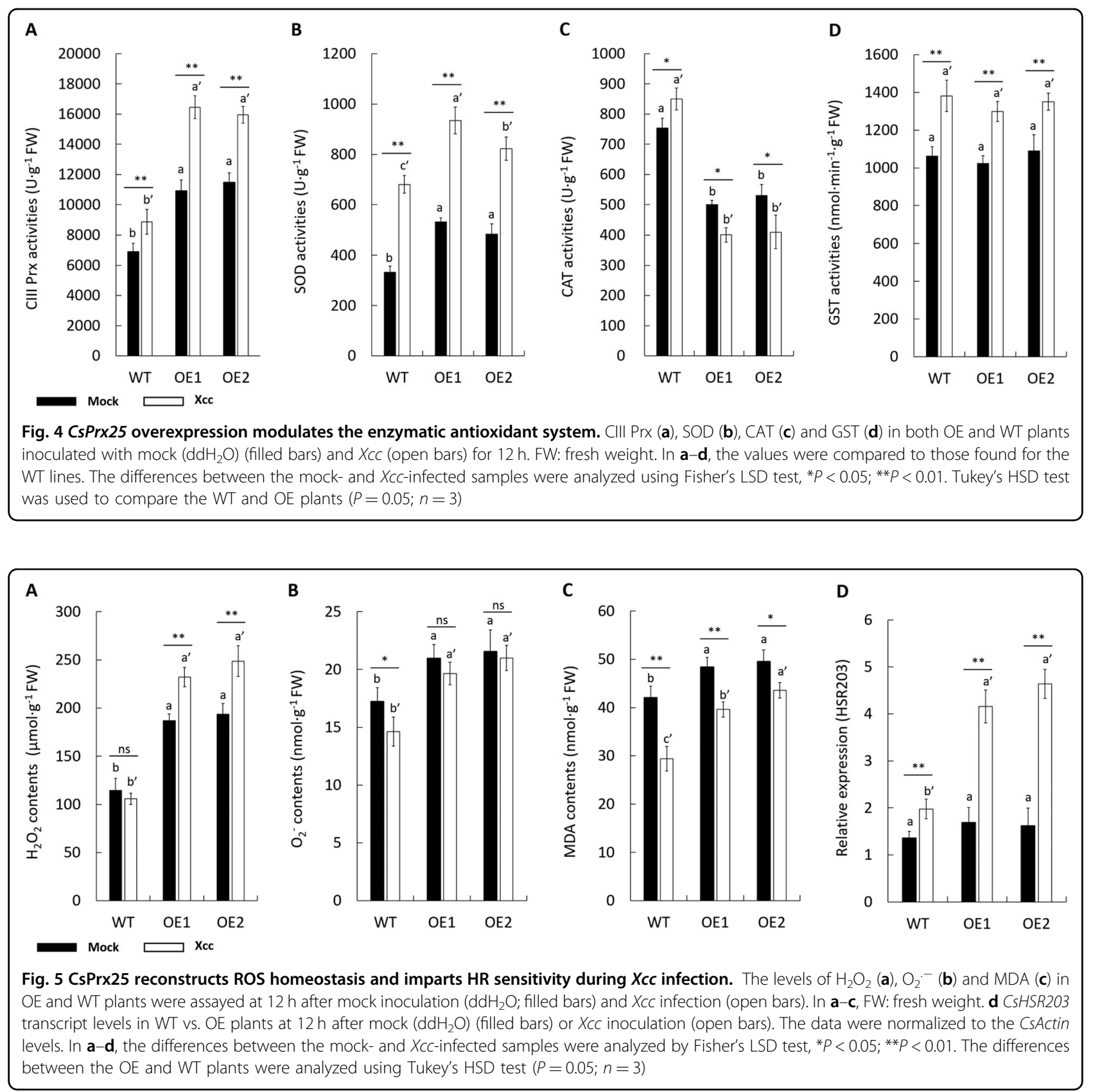

revealed elevated levels of MDA, and these levels were modestly reduced in response to $X c c$ infection (Fig. 5c), which indicate lower levels of damage following Xcc infection in both the transgenic and WT plants. These data indicate that Wanjincheng has the ability to suppress the oxidative damage caused by $X c c$ infection, and this suppression is strengthened by $C s \operatorname{Prx} 25$ overexpression. $\mathrm{H}_{2} \mathrm{O}_{2}$ is a key mediator of an early $\mathrm{HR}$. Because CsPrx 25 overexpression regulates $\mathrm{H}_{2} \mathrm{O}_{2}$ modulation, the immediate question was whether the HR is also altered in the transgenic plants. To investigate the relationship between the increased $\mathrm{CBC}$ resistance induced by CsPrx 25 and HR, we assessed the HR of the transgenic plants before and after $X c c$ infection. The expression of the HR marker gene, CsHSR203 ${ }^{56-58}$ was significantly upregulated in the transgenic plants infected with $X c c$ but only modestly increased in the Xccinfected WT plants. No obvious changes in the expression of CsHSR2O3 were observed between the transgenic and WT plants in the absence of Xcc infection (Fig. 5d). We therefore conclude that the transgenic plants are more sensitive to a HR following Xcc infection, which increases the early resistance of transgenic plants to $\mathrm{CBC}$. 


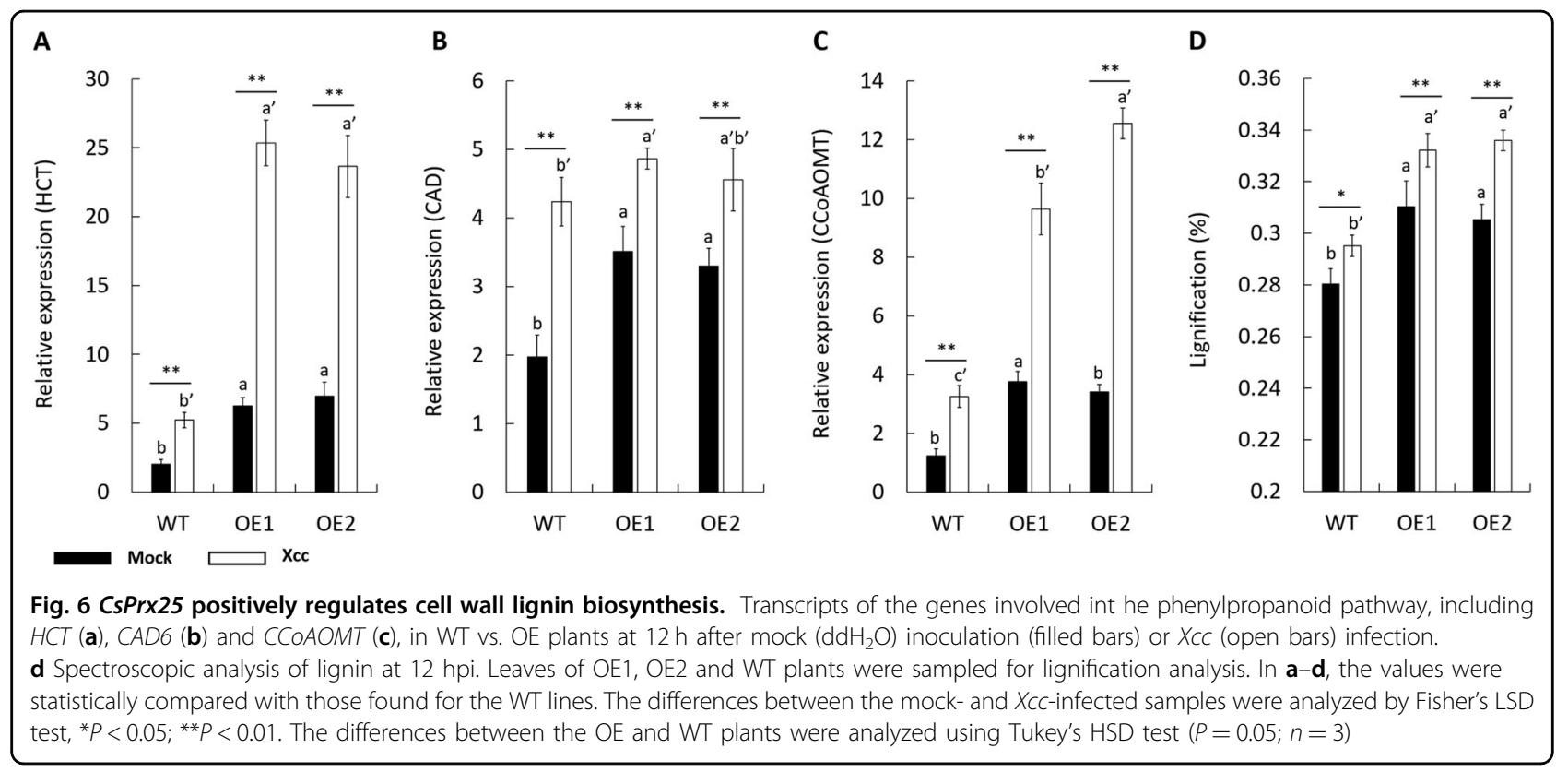

\section{CsPrx25 overexpression enhances lignification as an apoplastic barrier for Xcc infection}

CIII Prxs regulate cell wall lignification, which suggests a direct role of CIII Prxs on the cell walls of plants, ${ }^{6,59-61}$. To investigate the effects of lignification on $X c c$ resistance in transgenic plants, the role of CsPrx 25 in lignification was assessed. The transcript levels of lignin biosynthetic genes, namely, hydroxycinnamoyl transferase (CsHCT, CAP ID: Cs1g14450), cinnamyl alcohol dehydrogenase (CsCAD, CAP ID: Cs1g20590) and caffeoyl-CoA Omethyltransferase (CsCCoAOMT, CAP ID: Cs4g13430), were elevated in the leaves of the transgenic lines after mock inoculation and Xcc infection (Fig. 6a-c). These findings highlight the role of CsPrx25 in lignin biosynthesis. All the data were confirmed through lignin assays, which showed higher values in the transgenic compared with the WT plants (Fig. 6d). These data therefore reflect the role of CsPrx25 in the polymerization of lignin during its biosynthesis and highlight its importance in $\mathrm{CBC}$ resistance through enhanced lignification.

\section{CsPrx25 enhances CBC resistance, and this effect is associated with ROS homeostasis reconstruction and lignification}

CsPrx 25 overexpression confers ROS homeostasis to the transgenic lines through modulation of the enzymatic antioxidant system (Figs. 4-5). The levels of lignin were also higher in the transgenic lines than in the WT plants, and some lignin biosynthetic genes were more highly expressed in the transgenic lines (Fig. 6). Based on these results, we proposed a model to explain how Calamondin and CsPrx 25-OE transgenic Wanjincheng acquired CBC resistance (Fig. 7). In Calamondin, Xcc infection improves

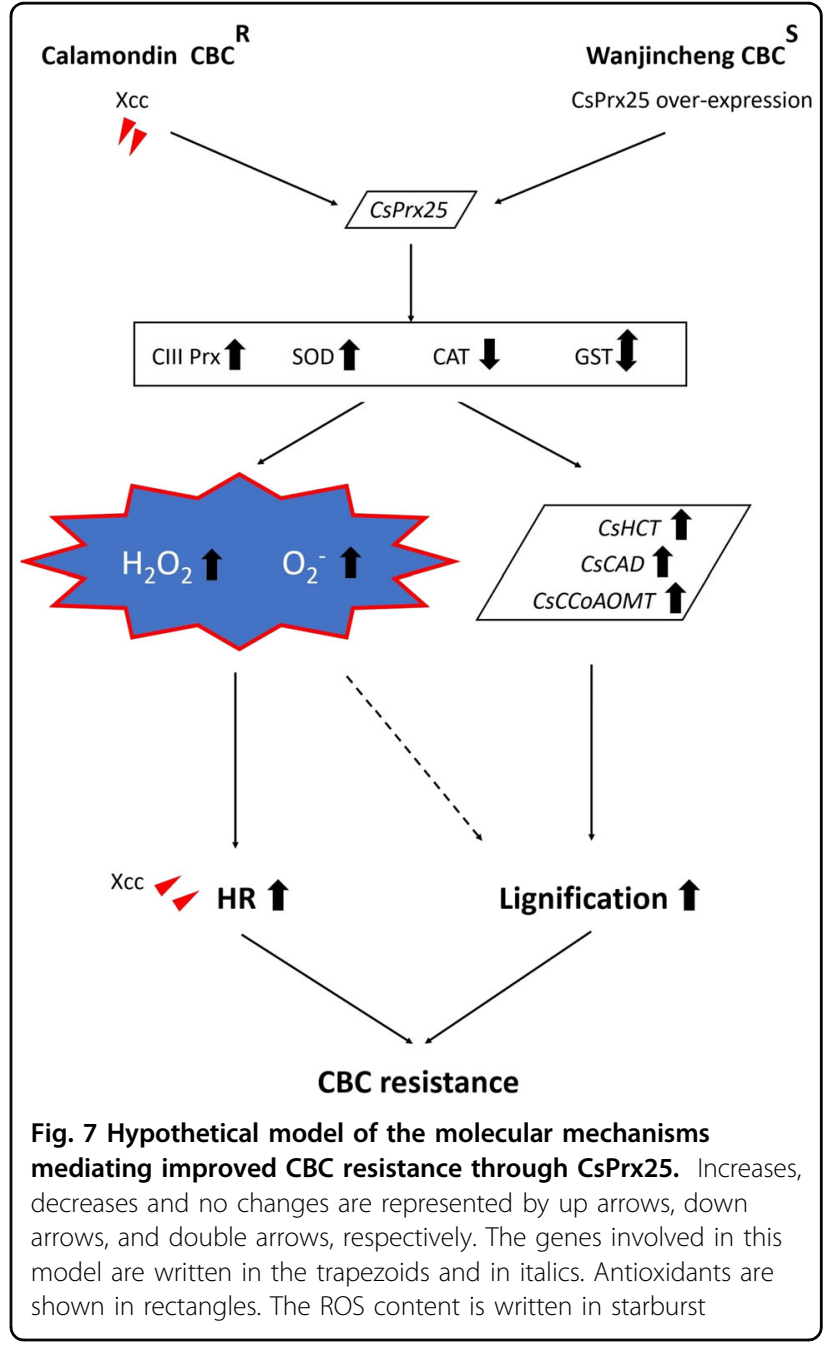


the levels of CsPrx 25, and this effect enhances the $\mathrm{H}_{2} \mathrm{O}_{2}$ levels and $H R$ sensitivity and induces lignification, resulting in $\mathrm{CBC}$ resistance. The overexpression of CsPrx 25 in CBC-susceptible Wanjincheng establishes ROS homeostasis, and higher $\mathrm{H}_{2} \mathrm{O}_{2}$ levels confer $\mathrm{HR}$ sensitivity in response to $\mathrm{Xcc}$ infection. In the transgenic plants, CsPrx 25 overexpression also enhanced lignin biosynthesis, reinforcing the apoplastic barrier for Xcc infection. Through these two mechanisms, CsPrx25 promotes $\mathrm{CBC}$ resistance.

\section{Materials and methods}

\section{Plants, bacteria and growth conditions}

All the plants were obtained from the National Citrus Germplasm Repository. Wanjincheng (C. sinensis) was used for gene transformations. All the plants were grown at $28^{\circ} \mathrm{C}$ in a greenhouse. The $X c c$ variants were derived from citrus leaves that are susceptible to natural infections. The $X c c$ cultures were grown at $28^{\circ} \mathrm{C}$ in peptoneyeast extract-malt extract containing $1.5 \% \quad(\mathrm{w} / \mathrm{v}) \quad \mathrm{D}-$ glucose.

\section{In silico characterization of CsPrx25}

The complete transcript sequence of CsPrx25 was amplified from Wanjincheng leaves using the primers $\mathrm{F}_{\text {clone }}$ (ATGGCAACTGCTTCAGCTTCT) and $\mathrm{R}_{\text {clone }}$ (TTAGATAATCCCAGACCAAGC). PeroxiScan was used for the family classification of CsPrx $25^{20}$. Blast tools built in RedoxiBase ${ }^{20,44}$, CAP $^{46}$, CitGVD $^{62}$ and SMART ${ }^{63}$ were used to reconfirm the sequence of CsPrx 25 retrieved by PCR. The chromosomal loci and the locations of exons and introns were defined using GSDS V2.0 $0^{64}$ based on the genome assembly of C. sinensis in CAP. SignalP V4.0 ${ }^{65}$ was used for signal peptide predictions, and CELLO V2. $5^{66}$ was used for cellular localization prediction. Phyre V2. ${ }^{67}$ was used for the 3D assessments of CsPrx25. The gene, protein and coding sequences (CDSs) of CsPrx25 are shown in Table S2.

\section{Transient expression of GFP-tagged CsPrx25}

The coding sequence (CDS) of CsPrx25 lacking a stop codon was amplified with flanking restriction sites using the primers $\mathrm{F}_{\mathrm{SC}}$ (CGGGGTACCATGGCTGTTCATCAA CATTATCTGG) (KpnI) and $\mathrm{R}_{\mathrm{SC}}$ (TCCCCCGGGTCAC TGGTTTGAAATTAAAGGATCT) (SmaI), digested, recovered and cloned into pLGNe-GFP driven by the $35 \mathrm{~S}$ promoter to construct the recombinant plasmid pLGNeCsPrx 25-GFP. The pLGNe-CsPrx25-GFP plasmid encodes a fusion protein composed of CsPrx 25 and GFP. The plasmids were heat-shocked into Agrobacterium EHA105. The transformed EHA105 was infiltrated into onion epidermal cells, and the GFP fluorescence signals were observed at $48 \mathrm{hpi}$ by laser-scanning confocal microscopy (LSM 510 Meta, Zeiss).

\section{Treatments with $X c c$ and phytohormones}

The expression of CsPrx 25 in excised leaves maintained in culture plates for $16 \mathrm{~h}$ of light and $8 \mathrm{~h}$ of darkness was assessed. Diluted Xcc (OD600: 0.8) was inoculated onto the leaves at $28^{\circ} \mathrm{C}$, and after defined durations, the expression of CsPrx 25 was assessed by qRT-PCR. For phytohormone assessments, leaf discs were soaked in $10 \mu \mathrm{mol} \mathrm{L}^{-1}$ SA or $100 \mu \mathrm{mol} \mathrm{L}^{-1}$ MeJA and collected for qRT-PCR assays of exogenous phytohormones. The primers used for CsPrx 25 detection were $\mathrm{F}_{\mathrm{RT}}$ (CCCCACT TCGGATTCCAACA) and $\mathrm{R}_{\mathrm{RT}}$ (CAACCCCTGTCGGTT CATCA).

\section{Overexpression vector construction and plant transformation}

For the generation of overexpression lines, full-length CsPrx 25 was PCR amplified using $\mathrm{F}_{\mathrm{OEC}}$ (GGGGTACCA TGGCAACTGCTTCAGCTTC) and R OEC (CGGGATC CTTAGATAATCCCAGACCAAGCC) and cloned into pLGNe to yield the recombinant plasmid pLGNeCsPrx25. Wanjincheng shoot transformations were performed using Agrobacterium tumefaciens as previously described by $\mathrm{Li}$ and $\mathrm{He}^{48,50}$.

\section{Validation of the transgenic lines by PCR and GUS assays}

PCR assays were used to confirm the presence of the transgenic gene with the primers $\mathrm{F}_{\mathrm{OED}}$ (CGACACGCT TGTCTACTCCA) and R $\mathrm{OED}$ (CGGGATCCTTAGATA ATCCCAGACCAAGCC). GUS activity was assessed through histochemical analysis ${ }^{48,51}$.

\section{Southern blot assay}

Total genomic DNA (gDNA) was extracted from the leaves of the transgenic plants and WT plants using a CTAB kit (Zoonbio, China). The gDNA was fragmented using the restriction enzyme EcoRI, and the DNA fragments were separated on a $0.7 \%$ agarose gel and transferred to a Hybond- $\mathrm{N}^{+}$membrane (Amersham, UK). The NPTII coding gene labeled by digoxin (DIG) was used to hybridize the membrane-bound DNA (Roche, Switzerland). The nylon membrane was then exposed using nonradioactive probe detection. In the Southern blot assay, the pLGNe-CsPrx 25 plasmid was used as the positive control.

\section{Assessment of $\mathrm{CBC}$ resistance}

$\mathrm{CBC}$ resistance analyses were performed as previously described $^{48,68,69}$. Briefly, six punctures were made in six healthy mature leaves of each transgenic line via $0.5-\mathrm{mm}$ pins, and $1 \mu \mathrm{L}$ of each $X c c$ suspension $\left(1 \times 10^{5} \mathrm{cfu} \mathrm{mL}^{-1}\right)$ was subsequently inoculated. $\mathrm{CBC}$ development was assessed at $10 \mathrm{dpi}$, and both the disease severity (DS) and lesion size (LS) of the diseased spots were used for the assessment of CBC resistance. The DS was calculated as 
previously described ${ }^{51,70}$. CBC resistance was further evaluated through $X c c$ infiltration assays $\left(1 \times 10^{5} \mathrm{cfu} \mathrm{mL}^{-}\right.$ ${ }^{1}$ ), and canker symptoms were imaged at $10 \mathrm{dpi}$.

\section{Biochemical analysis}

The activities of CIII Prx, SOD, CAT and GST and the concentrations of $\mathrm{H}_{2} \mathrm{O}_{2}, \mathrm{O}_{2}{ }^{-}$, MDA, and lignin were measured via SinoBestBio assays (Shanghai, China). The experiments were repeated three times, and the results are shown as the means \pm SEs.

\section{RNA isolation, CDNA synthesis and qRT-PCR assay}

Miniprep kits (AidLab) were used for RNA isolation, and cDNA was synthesized using TaKaRa kits. qRT-PCR was performed using QuantStudio 7. The values were normalized to the CsActin levels (GenBank accession: GU911361.1, CAP ID: Cs1g05000) obtained using $\mathrm{F}_{\text {Actin }}$ (CATCCCTCAGCACCTTCC) and $\mathrm{R}_{\text {Actin }}$ (CCAACCT TAGCACTTCTCC). The qRT-PCR parameters were as follows: $95^{\circ} \mathrm{C}$ for 5 min followed by 40 cycles of $95^{\circ} \mathrm{C}$ for $10 \mathrm{~s}$ and $56^{\circ} \mathrm{C}$ for $30 \mathrm{~s}$. The reaction mixtures (total volume of $12 \mu \mathrm{L}$ ) contained $50 \mathrm{ng}$ of cDNA, $0.5 \mu \mathrm{M}$ primers and $6 \mu \mathrm{L}$ of the PCR mix. The relative gene expression levels were assessed using the $2^{-\Delta \Delta C T}$ method $^{71}$. NCBI was used for qRT-PCR primer design (Supplementary Table S3). The data are presented as the means from three independent biological repeats.

\section{Statistics}

The data were analyzed using SPSS V22. Gene expression was compared by analysis of variance (ANOVA). The statistical significance was analyzed by Fisher's LSD test. ${ }^{*} P<0.05$ and ${ }^{* *} P<0.01$ indicate significant and extremely significant differences, respectively. The plant lines were compared using Tukey's HSD test $(P=0.05)$.

\section{Discussion}

CIII Prxs belong to a plant-specific multigene family that promotes disease resistance ${ }^{18,33,34}$, lignification, the flexibility of cell walls and suberization ${ }^{29,30}$. In sweet orange, 72 CIII Prxs have been identified ${ }^{28}$. The expression of each isoform varies across tissues and can be influenced by environmental factors, which suggests that different peroxidase isoenzymes regulate distinct processes $^{72}$. The distribution of enzymes to either the cell walls or vacuoles and their destinations reflect their specific functions ${ }^{31}$. In CBC-resistant and $\mathrm{CBC}$-susceptible varieties, CsPrx 25 exhibits altered expression patterns (Fig. 2d-f), which suggests its role during CBC development. The importance of CIII Prxs for the resistance of plants to pathogenic diseases was identified through reverse genetics. CIII Prxs mediate innate resistance both passively and actively ${ }^{6}$. HvPrx $40^{40}$ and TaPrx10 39,41 enhance the resistance of wheat against wheat powdery mildew. Here, CsPrx25 was found to mediate protection against Xcc pathogenesis, which confirmed its role as a CIII Prx and further highlighted the importance of this family in pathogen immunity in sweet orange. We explored its functional role using overexpression strategies and found that CsPrx25 strongly conferred $\mathrm{CBC}$ resistance to the transgenic plants (Fig. $3 g-j$ ).

Oxidative bursts, particularly the production of $\mathrm{H}_{2} \mathrm{O}_{2}$ and $\mathrm{O}_{2}{ }^{-}$, are common innate responses in plant cells in response to pathogen infection ${ }^{38}$. As key enzymes for ROS homeostasis in plants, CIII Prxs have multiple functions and are proposed to serve as key regulators of the extracellular $\mathrm{H}_{2} \mathrm{O}_{2}$ and $\mathrm{O}_{2}{ }^{-}$levels depending on peroxidative cycles (ROS scavenging) or hydroxylic cycles (ROS production $)^{73}$. Plant defense responses are governed by the ROS levels and peroxidase-generated radicals, which mediate cell wall reinforcement, damage repair ${ }^{4,6}$ and apoptotic responses to induce plant resistance ${ }^{5,6}$. In this study, the molecular mechanisms of CsPrx25 were explored. Based on our analysis of ROS homeostasis and enzymatic antioxidant activities in the transgenic plants, we concluded that CsPrx 25 overexpression enhances CIII Prx activities and leads to a simultaneous improvement in the $\mathrm{H}_{2} \mathrm{O}_{2}$ and $\mathrm{O}_{2}{ }^{-}$content (Fig. $5 \mathrm{a}, \mathrm{b}$ ). In plants, the $\mathrm{HR}$ is directly related to plant disease resistance and represents the classic response to pathogen infection ${ }^{6}$. These reactions lead to both rapid and localized necrosis of the infected tissues and thus prevent the spread of infection $^{56,57} \cdot \mathrm{H}_{2} \mathrm{O}_{2}$ is key to the HR and is related to programmed cell death (PCD) in infected plants ${ }^{58}$. To investigate the relationship between the $\mathrm{CBC}$ resistance induced by CsPrx 25 and the HR, we assessed the HR of the transgenic plants before and after Xcc infection (Fig. 5d). HSR203 is upregulated by plant HRs and is used as a marker for the HR levels ${ }^{56,58}$. In this study, the links among CsPrx 25 activity, ROS content and HR level were established. Cell wall lignification was further shown to mediate $\mathrm{CBC}$ resistance, which was also demonstrated in rice due to the enhancement in Xanthomonas oryzae resistance conferred by CIII Prx-mediated lignification ${ }^{73}$.

Due to the evolutionary diversity and functional diversity of CIII Prxs, different studies have drawn different links between CIII Prx and disease resistance. Increased LePrx06 makes tomato more susceptible to Pseudomonas syringae infection. In contrast to CsPrx 25, the suppression of LePrx06 can enhance resistance to this pathogen ${ }^{74}$. Long-term studies of the relationship between the ROS levels and the development of $\mathrm{CBC}$ have revealed increased peroxidase activity and thus a reduced ROS content. Furthermore, the reduction in the ROS levels was associated with $\mathrm{CBC}$ resistance. These effects parallel the overexpression of MdATG18a and can enhance resistance to Diplocarpon mali infection via $\mathrm{H}_{2} \mathrm{O}_{2}$ scavenging ${ }^{75}$. Cybrids of grapefruit with a kumquat plastid genome 
exhibit increased $\mathrm{CBC}$ resistance through an early upregulation of ROS-controlling genes upon Xcc infection ${ }^{76}$. These findings illustrate potential links between ROS homeostasis mediated by plastid ROS-controlling genes and $X c c$ resistance. However, this study revealed that CsPrx25 is an apoplast-localized enzyme rather than a plastid enzyme (Fig. 2b, c), and this knowledge expands the list of ROS-controlling enzymes that can upregulate $\mathrm{CBC}$ resistance.

In this study of CsPrx25, regulation of the ROS levels by CsPrx25 and improvements in HR sensitivity were the major mechanisms through which transgenic citrus developed resistance to CBC. Although CsPrx25 overexpression greatly improved the resistance of Wanjincheng to CBC, CsPrx25-overexpressing Wanjincheng cells were still not as resistant as Calamondin cells, which might be due to the fact that Calamondin also has other mechanisms to maintain an even higher level of CBC resistance. Anyway, this study explores new insights into the mechanisms of CIII Prxs in CBC resistance and provides potential clues for breeding $\mathrm{CBC}$-resistant citrus.

\section{Acknowledgements}

The National Key Research and Development Program of China (2018YFD1000306), the Natural Science Foundation of Chongqing (cstc2020jcyj-msxmX1064), the Fundamental Research Funds for the Central Universities (SWU115025), the Earmarked Funds for the China Agriculture Research System (CARS-26), and the Key Project of Guangxi Science and Technology (GuiKeAA18118046-6) funded this study.

\section{Author details \\ ${ }^{1}$ Citrus Research Institute, Southwest University/Chinese Academy of Agricultural Sciences, Chongqing 400712, China. 'Laboratoire de Recherche en Sciences Végétales, Université de Toulouse, CNRS, UPS, Auzeville-Tolosane 31320, France. ${ }^{3}$ Key Laboratory of Plant Hormones and Development Regulation of Chongqing, School of Life Sciences, Chongqing University, 401331 Chongqing, China}

\section{Author contributions}

Q.L. and S.C. conceived the experiments; Q.L., J.Q., X.Q. and W.D. conduced the experiments; Q.L. and C.D. conducted the bioinformatics analysis; Q.L. and W.D. were responsible for the data analyses; and Q.L. and Y.H. wrote the paper.

\section{Conflict of interest}

The authors declare that they have no conflict of interest.

Supplementary Information accompanies this paper at (https://doi.org/ 10.1038/s41438-020-00415-9).

Received: 20 March 2020 Revised: 2 September 2020 Accepted: 4 September 2020

Published online: 01 December 2020

\section{References}

1. Molina, L. \& Kahmann, R. An ustilago maydis gene involved in $\mathrm{H} 2 \mathrm{O} 2$ detoxification is required for virulence. Plant Cell 19, 2293-2309 (2007).

2. Peters, L. P. et al. Functional analysis of oxidative burst in sugarcane smutresistant and -susceptible genotypes. Planta 245, 749-764 (2017).

3. Pitino, M., Armstrong, C. M. \& Duan, Y. Rapid screening for citrus canker resistance employing pathogen-associated molecular pattern-triggered immunity responses. Hortic. Res. 2, 15042 (2015).
4. Ostergaard, L. et al. Expression and high-resolution structure of a plant peroxidase with implications for lignification. Plant Mol. Biol. 44, 231-243 (2000).

5. Passardi, F., Penel, C. \& Dunand, C. Performing the paradoxical: how plant peroxidases modify the cell wall. Trends Plant Sci. 9, 534-540 (2004).

6. Schweizer, P. Tissue-specific expression of a defence-related peroxidase in transgenic wheat potentiates cell death in pathogen-attacked leaf epidermis. Mol. Plant Pathol. 9, 45-57 (2008).

7. Liu, G. et al. Profiling of wheat class III peroxidase genes derived from powdery mildew-attacked epidermis reveals distinct sequence-associated expression patterns. Mol. Plant Microbe Interact. 18, 730-741 (2005).

8. Han, F. P., Fedak, G., Ouellet, T., Dan, H. \& Somers, D. J. Mapping of genes expressed in Fusarium graminearum-infected heads of wheat cultivar 'Frontana'. Genome 48, 88-96 (2005).

9. Mittler, R. et al. Transgenic tobacco plants with reduced capability to detoxify reactive oxygen intermediates are hyperresponsive to pathogen infection. Proc. Natl Acad. Sci. USA 96, 14165-14170 (1999).

10. Barna, B., Fodor, J., Harrach, B. D., Pogány, M. \& Király, Z. The Janus face of reactive oxygen species in resistance and susceptibility of plants to necrotrophic and biotrophic pathogens. Plant Physiol. Biochem. 59, 37-43 (2012).

11. Apel, K. \& Hirt, H. Reactive oxygen species: metabolism, oxidative stress, and signal transduction. Annu. Rev. Plant Biol. 55, 373-399 (2004).

12. Torres, M. A. ROS in biotic interactions. Physiol. Plant 138, 414-429 (2010).

13. Aviello, G. \& Knaus, U. G. NADPH oxidases and ROS signaling in the gastrointestinal tract. Mucosal Immunol. 11, 1011-1023 (2018).

14. Peters, L. P. et al. Differential responses of the antioxidant system of ametryn and clomazone tolerant bacteria. PLoS ONE 9, e112271 (2014).

15. Pieterse, C. M., Leon-Reyes, A., Van der Ent, S. \& Van Wees, S. C. Networking by small-molecule hormones in plant immunity. Nat. Chem. Biol. 5, 308-316 (2009).

16. Thomma, B. P. et al. Separate jasmonate-dependent and salicylate-dependent defense-response pathways in Arabidopsis are essential for resistance to distinct microbial pathogens. Proc. Natl Acad. Sci. USA 95, 15107-15111 (1998).

17. Pieterse, C. M. et al. Hormonal modulation of plant immunity. Annu. Rev. Cell Dev. Biol. 28, 489-521 (2012).

18. Li, Q. et al. Explosive tandem and segmental duplications of multigenic families in Eucalyptus grandis. Genome Biol. Evol. 7, 1068-1081 (2015).

19. Li, Q., San Clemente, H., He, Y. R., Fu, Y. Y. \& Dunand, C. Global evolutionary analysis of 11 gene families part of reactive oxygen species (ROS) gene network in four Eucalyptus species. Antioxidants 9, 19 (2020).

20. Savelli, B. et al. RedoxiBase: a database for ROS homeostasis regulated proteins. Redox Biol. 26, 101247 (2019).

21. Mbadinga Mbadinga, D., Li, Q., Ranocha, P., Martinez, Y. \& Dunand, C. Global analysis of non-animal peroxidases provides insights into the evolution of this gene family in the green lineage. J. Exp. Bot. 71, 3350-3360 (2020).

22. Tognolli, M., Penel, C., Greppin, H. \& Simon, P. Analysis and expression of the class III peroxidase large gene family in Arabidopsis thaliana. Gene 288, 129-138 (2002).

23. Cosio, C. \& Dunand, C. Transcriptome analysis of various flower and silique development stages indicates a set of class III peroxidase genes potentially involved in pod shattering in Arabidopsis thaliana. BMC Genom. 11, 528 (2010).

24. Passardi, F., Longet, D., Penel, C. \& Dunand, C. The class III peroxidase multigenic family in rice and its evolution in land plants. Phytochemistry $\mathbf{6 5}$, 1879-1893 (2004).

25. Yan, J. et al. Genome-wide and evolutionary analysis of the class III peroxidase gene family in wheat and Aegilops tauschii reveals that some members are involved in stress responses. BMC Genom. 20, 666 (2019).

26. Ren, L. L. et al. Subcellular relocalization and positive selection play key roles in the retention of duplicate genes of populus class III peroxidase family. Plant Cell 26, 2404-2419 (2014).

27. Cao, Y. et al. Structural, evolutionary, and functional analysis of the class III peroxidase gene family in chinese pear. Front. Plant Sci. 7, 1874 (2016).

28. Li, Q. et al. Genomewide analysis of the CIII peroxidase family in sweet orange (Citrus sinensis) and expression profiles induced by Xanthomonas citri subsp. citri and hormones. J. Genet. 99, 13 (2020).

29. Fernández-Pérez, F., Pomar, F., Pedreño, M. A. \& Novo-Uzal, E. Suppression of arabidopsis peroxidase 72 alters cell wall and phenylpropanoid metabolism. Plant Sci. 239, 192-199 (2015).

30. Pandey, V. P. \& Dwivedi, U. N. A ripening associated peroxidase from papaya having a role in defense and lignification: heterologous expression and insilico and in-vitro experimental validation. Gene 555, 438-447 (2015).

31. Shigeto, J. \& Tsutsumi, Y. Diverse functions and reactions of class III peroxidases. N. Phytol. 209, 1395-1402 (2016). 
32. Passardi, F., Cosio, C., Penel, C. \& Dunand, C. Peroxidases have more functions than a swiss army knife. Plant Cell Rep. 24, 255-265 (2005).

33. Liszkay, A., Kenk, B. \& Schopfer, P. Evidence for the involvement of cell wall peroxidase in the generation of hydroxyl radicals mediating extension growth. Planta 217, 658-667 (2003).

34. Mclnnis, S. M., Desikan, R., Hancock, J. T. \& Hiscock, S. J. Production of reactive oxygen species and reactive nitrogen species by angiosperm stigmas and pollen: potential signalling crosstalk? N. Phytol. 172, 221-228 (2006).

35. Mei, W., Qin, Y., Song, W., Li, J. \& Zhu, Y. Cotton GhPOX1 encoding plant class III peroxidase may be responsible for the high level of reactive oxygen species production that is related to cotton fiber elongation. J. Genet. Genomics 36 141-150 (2009).

36. Cao, J., Jiang, M., Li, P. \& Chu, Z. Genome-wide identification and evolutionary analyses of the PP2C gene family with their expression profiling in response to multiple stresses in Brachypodium distachyon. BMC Genom. 17, 175 (2016).

37. Van Loon, L. C., Rep, M. \& Pieterse, C. M. Significance of inducible defenserelated proteins in infected plants. Annu. Rev. Phytopathol. 44, 135-162 (2006).

38. Almagro, L. et al. Class III peroxidases in plant defence reactions. J. Exp. Bot. 60, 377-390 (2009).

39. Radwan, M. A., El-Gendy, K. S. \& Gad, A. F. Biomarkers of oxidative stress in the land snail, Theba pisana for assessing ecotoxicological effects of urban metal pollution. Chemosphere 79, 40-46 (2010).

40. Johrde, A. \& Schweizer, P. A class III peroxidase specifically expressed in pathogen-attacked barley epidermis contributes to basal resistance. Mol. Plant Pathol. 9, 687-696 (2008).

41. Altpeter, F. et al. Stable expression of a defense-related gene in wheat epidermis under transcriptional control of a novel promoter confers pathogen resistance. Plant Mol. Biol. 57, 271-283 (2005).

42. Omar, A. A., Murata, M. M., El-Shamy, H. A., Graham, J. H. \& Grosser, J. W. Enhanced resistance to citrus canker in transgenic mandarin expressing Xa21 from rice. Transgenic Res 27, 179-191 (2018).

43. Schaad, N. W. et al. Reclassification of Xanthomonas campestris pv. citri (ex Hasse 1915) Dye 1978 forms A, B/C/D, and E as X. smithii subsp. citri (ex Hasse) sp. nov. nom. rev. comb. nov., X. fuscans subsp. aurantifolii (ex Gabriel 1989) sp. nov. nom. rev. comb. nov., and X. alfalfae subsp. citrumelo (ex Riker and Jones) Gabriel et al., 1989 sp. nov. nom. rev. comb. nov; X. campestris pv malvacearum (ex smith 1901) Dye 1978 as X. smithii subsp. smithii nov. comb. nov. nom. nov.; X. campestris pv. alfalfae (ex Riker and Jones, 1935) dye 1978 as X. alfalfae subsp. alfalfae (ex Riker et al., 1935) sp. nov. nom. rev.; and "var. fuscans" of X. campestris pv. phaseoli (ex Smith, 1987) Dye 1978 as X. fuscans subsp. fuscans sp. nov. Syst. Appl. Microbiol. 28, 494-518 (2005).

44. Fawal, N. et al. PeroxiBase: a database for large-scale evolutionary analysis of peroxidases. Nucleic Acids Res. 41, D441-D444 (2013).

45. Savelli, B. et al. RedoxiBase: A database for ROS homeostasis regulated proteins. Redox Biol. 26, 5 (2019).

46. Wang, J. et al. Citrus sinensis annotation project (CAP): a comprehensive database for sweet orange genome. PLoS ONE 9, e87723 (2014).

47. Welinder, K. G. et al. Structural diversity and transcription of class III peroxidases from Arabidopsis thaliana. Eur. J. Biochem. 269, 6063-6081 (2002).

48. He, Y. et al. Functional analysis of citrus AP2 transcription factors identified CSAP2-09 involved in citrus canker disease response and tolerance. Gene 707, 178-188 (2019).

49. Zuo, W. et al. A maize wall-associated kinase confers quantitative resistance to head smut. Nat. Genet. 47, 151-157 (2015).

50. Li, Q. et al. CSWAKL08, a pathogen-induced wall-associated receptor-like kinase in sweet orange, confers resistance to citrus bacterial canker via ROS control and JA signaling. Hort. Res. 7, 15 (2020).

51. Sendín, L. N. et al. Inducible expression of Bs2 R gene from Capsicum chacoense in sweet orange (Citrus sinensis L. Osbeck) confers enhanced resistance to citrus canker disease. Plant Mol. Biol. 93, 607-621 (2017).

52. Du, X. M., Yin, W. X., Zhao, Y. X. \& Zhang, H. The production and scavenging of reactive oxygen species in plants. Sheng Wu Gong. Cheng Xue Bao 17, 121-125 (2001).
53. Hückelhoven, R. \& Kogel, K. H. Reactive oxygen intermediates in plant-microbe interactions: who is who in powdery mildew resistance? Planta $\mathbf{2 1 6}, \mathbf{8 9 1 - 9 0 2}$ (2003).

54. Soosaar, J. L., Burch-Smith, T. M. \& Dinesh-Kumar, S. P. Mechanisms of plant resistance to viruses. Nat. Rev. Microbiol. 3, 789-798 (2005).

55. Mishra, M. K. et al. Overexpression of WsSGTL1 gene of Withania somnifera enhances salt tolerance, heat tolerance and cold acclimation ability in transgenic Arabidopsis plants. PLoS ONE 8, e63064 (2013).

56. Pontier, D., Tronchet, M., Rogowsky, P., Lam, E. \& Roby, D. Activation of hsr203, a plant gene expressed during incompatible plant-pathogen interactions, is correlated with programmed cell death. Mol. Plant Microbe Interact. 11, 544-554 (1998).

57. Pontier, D., Godiard, L., Marco, Y. \& Roby, D. Hsr203J, a tobacco gene whose activation is rapid, highly localized and specific for incompatible plant/ pathogen interactions. Plant J. 5, 507-521 (1994).

58. Tronchet, M., Ranty, B., Marco, Y. \& Roby, D. Hsr203 antisense suppression in tobacco accelerates development of hypersensitive cell death. Plant J. 27, 115-127 (2001)

59. Herrero, J. et al. Bioinformatic and functional characterization of the basic peroxidase 72 from Arabidopsis thaliana involved in lignin biosynthesis. Planta 237, 1599-1612 (2013).

60. Hiraga, S., Sasaki, K., Ito, H., Ohashi, Y. \& Matsui, H. A large family of class III plant peroxidases. Plant Cell Physiol. 42, 462-468 (2001).

61. Elfstrand, M., Sitbon, F., Lapierre, C., Bottin, A. \& von Arnold, S. Altered lignin structure and resistance to pathogens in spi 2-expressing tobacco plants. Planta 214, 708-716 (2002).

62. Li, Q. et al. CitGVD: a comprehensive database of citrus genomic variations. Hort. Res. 7, 12 (2020).

63. Letunic, I. \& Bork, P. 20 years of the SMART protein domain annotation resource. Nucleic Acids Res. 46, D493-D496 (2018).

64. Hu, B. et al. GSDS 2.0: an upgraded gene feature visualization server. Bioinformatics 31, 1296-1297 (2015).

65. Petersen, T. N., Brunak, S., von Heijne, G. \& Nielsen, H. SignalP 4.0: discriminating signal peptides from transmembrane regions. Nat. Methods 8, 785-786 (2011).

66. Yu, C. S., Chen, Y. C., Lu, C. H. \& Hwang, J. K. Prediction of protein subcellular localization. Proteins 64, 643-651 (2006).

67. Kelley, L. A., Mezulis, S., Yates, C. M., Wass, M. N. \& Sternberg, M. J. The Phyre2 web portal for protein modeling, prediction and analysis. Nat. Protoc. 10, 845-858 (2015).

68. Li, Q. et al. CsBZIP40, a BZIP transcription factor in sweet orange, plays a positive regulatory role in citrus bacterial canker response and tolerance. PloS ONE 14, e0223498 (2019).

69. Peng, A. et al. Engineering canker-resistant plants through CRISPR/Cas9-targeted editing of the susceptibility gene CSLOB1 promoter in citrus. Plant Biotechnol. J. 15, 1509-1519 (2017).

70. Li, Q. et al. Systematic analysis and functional validation of citrus XTH genes reveal the role of $\mathrm{CSXTH04}$ in citrus bacterial canker resistance and tolerance. Front. Plant Sci. 10, 1109 (2019).

71. Livak, K. J. \& Schmittgen, T. D. Analysis of relative gene expression data using real-time quantitative $P C R$ and the 2(-delta delta $C(T)$ ) method. Methods $\mathbf{2 5}$ 402-408 (2001).

72. Caruso, C. et al. A basic peroxidase from wheat kernel with antifungal activity. Phytochemistry 58, 743-750 (2001)

73. Kawano, T. Roles of the reactive oxygen species-generating peroxidase reactions in plant defense and growth induction. Plant Cell Rep. 21, 829-837 (2003).

74. Coego, A. et al. An Arabidopsis homeodomain transcription factor, OVEREXPRESSOR OF CATIONIC PEROXIDASE 3, mediates resistance to infection by necrotrophic pathogens. Plant Cell 17, 2123-2137 (2005).

75. Sun, $X$. et al. MdATG18a overexpression improves tolerance to nitrogen deficiency and regulates anthocyanin accumulation through increased autophagy in transgenic apple. Plant Cell Environ. 41, 469-480 (2018).

76. Murata, M. M. et al. Novel plastid-nuclear genome combinations enhance resistance to citrus canker in cybrid grapefruit. Front. Plant Sci. 9, 1858 (2018). 